\title{
3 Research Square

\section{An evaluation of the effectiveness of a telephone assessment and advice service within an ED Physiotherapy clinic: A single-site cohort study}

\section{Marie Kelly ( $\sim$ kellym19@tcd.ie)}

Mercy University Hospital, Physiotherapy Department, Cork, Ireland

Anna Higgins

Mercy University Hospital, Physiotherapy Department, Cork, Ireland

Adrian Murphy

2Mercy University Hospital, Emergency Department, Cork, Ireland

Karen McCreesh

3University of Limerick, School of Allied Health, Limerick, Ireland

\section{Research Article}

Keywords: Musculoskeletal, non-attendance, telephone triage, timely access, satisfaction

Posted Date: September 2nd, 2020

DOI: https://doi.org/10.21203/rs.3.rs-69131/v1

License: (c) (i) This work is licensed under a Creative Commons Attribution 4.0 International License.

Read Full License 


\section{Abstract}

\section{Background}

In response to issues with timely access for musculoskeletal physiotherapy, telephone assessment and advice services have been evaluated in primary care settings. It is unclear whether this service model can reduce wait times and non-attendance rates for Emergency Department (ED) physiotherapy, compared to usual care. A secondary aim was to evaluate service user acceptability.

\section{Methods}

This was a single-site cohort study that compares data on non-attendance rates, wait time to first physiotherapy contact and participant satisfaction between patients that opted for a service based on initial telephone assessment and advice, versus routine face-to-face appointments. 116 patients were referred for ED physiotherapy over the 3-month pilot at the ED and out-patient physiotherapy department, $X$, Ireland. 91 patients (78\%) opted for the telephone assessment and advice service, with $40 \%(n=36)$ contacting the service. 25 patients (22\%) opted for the face-to-face service. Data on non-attendance rates and wait time was gathered using the hospital data reporting system. Satisfaction data was collected on discharge using a satisfaction survey adapted from the General Practice Assessment Questionnaire. Independent-samples t-test or Mann Whitney U Test was utilised depending on the distribution of the data. For categorical data, Chi-Square tests were performed. A level of significance of $p \leq 0.05$ was set for this study.

\section{Results}

Those that contacted the telephone assessment and advice service had a significantly reduced wait time (median 6 days; 3 - 8 days) compared to those that opted for usual care (median 35 days; 19 - 39 days) $(p \leq 0.05)$. There was no significant between-group differences for non-attendance rates or satisfaction.

\section{Conclusion}

Given the faster access to ED physiotherapy, without compromising on service user satisfaction, this telephone assessment and advice service, which can be introduced rapidly, could be helpful in triaging referrals and minimising face-to-face consultations in line with COVID-19 recommendations. However, a large scale study is warranted to confirm these findings.

\section{Introduction}

Musculoskeletal conditions make the most significant contribution to the global burden of disability, with more than $20 \%$ of the world's population living with a painful musculoskeletal disorder [1]. Given our aging population [2], the burden of these conditions is expected to increase, placing further demands on limited healthcare resources. Emergency departments (EDs) are one of the main providers of treatment for musculoskeletal conditions, particularly non-traumatic neck and back pain, with early access to 
physiotherapy strongly advocated for within the Irish Health Service Executive National Emergency Medicine Programme [3]. Physiotherapy intervention within a five-day period following injury significantly reduces work absenteeism [4], and consequently, has economic benefits, with musculoskeletal conditions ranked as the second largest cause of days lost from work [5]. However, timely access to physiotherapy is often an issue, with waiting lists for treatment of several months in some regions [6] which is likely to result in adverse effects on health outcomes and increased healthcare utilisation for patients with musculoskeletal conditions [7]. Furthermore, delayed access to physiotherapy can lead to increased nonattendance rates, with many not attending appointments when they are finally offered one [8]. This, together with the fact that those that gain minimal or no benefit from physiotherapy might have benefited more if they have been reviewed more quickly $[9,10]$ clearly illustrate that a significant amount of physiotherapy services are utilised ineffectively and inefficiently. [10]

Telehealth, a subcategory of eHealth [11], is becoming increasing popular in an attempt to meet these challenges. Clinicians are utilizing innovative methods of delivering care, including telephone consulting, with physiotherapy-led telephone assessment and advice services established across many regions such as the UK [12] and Australia [13]. Typically, within a telephone advice and assessment service, service users are invited to telephone a senior physiotherapist for initial assessment and advice, which is followed up with posted relevant self-management resources and exercise leaflets. Alternatively, face-toface consultations are arranged if deemed necessary following the initial telephone assessment or if the patient's symptoms are not resolving after the initial advice [8]. This service model is in keeping with recommendations from physiotherapy associations worldwide due to the COVID-19 pandemic, that the majority of appointments are conducted remotely, minimising face-to-face sessions where possible [14].

Although robust research is lacking on the role of telephone assessment within the field of physiotherapy, evidence exists on the safety, clinical- and cost-effectiveness, along with patient acceptability within other clinical settings such as nurse telephone consultation for routine asthma review and in out of hours primary care [15-18]. The only high quality randomized controlled trial within physiotherapy to evaluate a telephone triage service ('PhysioDirect') was conducted within a primary care setting, reporting that the service was as clinically effective as usual face-to-face care, with regards to participants' physical functioning [12]. Shorter waiting times and reductions in non-attendance rates were also illustrated. Furthermore, a nested qualitative study [19] concluded that a telephone assessment and advice service was broadly acceptable to participants, due to more timely access to advice.

This patient care pathway reflects the evidence about the effectiveness of different modalities within physiotherapy for various conditions. For example, trials have found that a single session of advice from a physiotherapist is as effective as a course of physiotherapy for patients with back pain [20, 21], with research also advocating a single physiotherapy advice session for those with persistent acute whiplash symptoms [22]. Furthermore, physiotherapy-led advice and exercise are effective in knee pain [23-25]. Alternatively, for other presentations such as shoulder and neck pain, evidence exists suggesting that manual therapy as an adjunct to advice and exercise is more effective than exercise and advice alone [26-28]. Therefore, a care pathway, which provides assessment, advice and triage initially, while 
reserving more intensive (and expensive) treatments for those who do not improve, may be the most costeffective strategy. This care pathway would also limit face-to-face consultations, in line with COVID-19 related recommendations [14]. However, to date this model of service delivery has yet to be evaluated within either the Irish healthcare system or physiotherapy ED setting. Therefore, the main objective of this study was to evaluate whether a telephone assessment and advice service can reduce the wait time and non-attendance rate for physiotherapy compared to the usual care pathway. A secondary aim was to evaluate whether a telephone assessment and advice service is acceptable and satisfactory to service users.

\section{Methods}

\section{Study design:}

This study was a single-site cohort study with two parallel groups with recruitment between May and August 2018. Data collection was complete in May 2019. The comparison was between patients that opted for a service based on initial telephone assessment and advice, versus routine face-to-face appointments. This study design was utilised due to a consistently high non-attendance rate (approx. average $30 \%$ ) and some qualitative research nested within the 'PhysioDirect' study [19] indicating that telephone assessment and advice services are best placed alongside face-to-face services rather than as a replacement. This study was approved by the Clinical Research Ethics Committee of the $X$, Ireland and carried out in the ED and outpatient physiotherapy department at X Hospital, X, Ireland. All participants provided signed informed consent to participate in this study, which was carried out in accordance with the Declaration of Helsinki. The STROBE standardised reporting guidelines were followed in both the conduct and reporting of this research [29] (Additional File 1).

\section{Participants:}

All adults (aged $\geq 18$ years of age) were invited to participate in this study, if following their attendance at the X Hospital ED, physiotherapy was deemed appropriate by a member of the ED team (Consultant, Non-Consultant Hospital Doctor or Advanced Nurse Practitioner). Inclusion criteria were deliberately broad to maximize generalizability. Participants were excluded if they were unable to communicate in English via telephone or were referred with non-musculoskeletal problems.

\section{Procedures:}

All eligible participants were provided with a participant information leaflet in ED and the two treatment pathways were discussed by a member of the ED team, with the patient choosing based on their preference. The first treatment option was the physiotherapy telephone assessment and advice service, while the alternative was the usual care pathway i.e. appointment made for a face-to-face consultation. Patients that opted for the telephone assessment and advice service had their verbal consent noted during the first telephone consultation with another copy of the participant information leaflet, questionnaires, consent form and prepaid return envelope sent out in the post on discharge. Those who 
did not respond to the first mail out were sent a second mail-out approx. two weeks later. Those that opted for a face-to-face consultation provided written informed consent during the first consultation if they wished to participate.

\section{The telephone assessment and advice service}

Patients were invited to telephone a senior physiotherapist at specific times for initial assessment and advice. Generally, at the end of the consultation, the senior physiotherapist posted a relevant advice leaflet about exercises and self-management to the patient and invited them to phone back in approx 2-4 weeks to report progress if appropriate. At that point, they were given further advice or booked for a faceto-face appointment if necessary. If the initial call indicated more urgent face-to-face care was required, this was booked at the outset.

\section{Usual care pathway}

Usual care generally involved an initial face-to-face physiotherapy assessment and then a series of follow-up treatment appointments over several weeks or months, according to therapist's discretion. .

\section{Data Collection}

To characterize the study population, demographic information such as employment status, location of symptoms, age, gender etc was recorded on a data collection form.

\section{Outcome measures}

The primary outcome measures were non-attendance rates, wait time to first physiotherapy contact and participant satisfaction. Non-attendance rates (defined as ratio of number of missed appointments to total number of scheduled appointments) and wait time to first physiotherapy contact was gathered using the hospital data reporting system, Implement Single Patient Administration System (iPIMS). Satisfaction data was collected using a satisfaction survey adapted from the General Practice Assessment Questionnaire, which has been utilised previously [12], with internal reliability confirmed using rotated factor analysis. Overall satisfaction with the service was based on one question. All questions use six point Likert scales. To characterize clinical outcome on the last physiotherapy appointment, both groups were asked one question either face-to-face or via telephone, about overall improvement in the main problem for which the patient was referred to physiotherapy (global improvement score - a seven point scale from "very much better" to "very much worse"). This was chosen as no disease specific measure would be appropriate for this study, given the varied range of musculoskeletal conditions referred to physiotherapy via ED.

\section{$\underline{\text { Statistical Analysis }}$}

All data analysis was undertaken using the Statistical Package for the Social Sciences Version (SPSS) 23.0 [30]. A level of significance of $p \leq 0.05$ was set for this study. Normality of the continuous variables 
was tested with the Shapiro-Wilk test and appropriate descriptive statistics were calculated. Where the normality assumption was violated, equivalent non-parametric tests were used. Analysis of primary and secondary outcomes was conducted on an intention to treat basis without imputation. The Mann Whitney U Test was utilized to evaluate between-group differences in wait time. Median and inter- quartile values (Q1 - Q3) are presented. Given the non-normal distribution these values are better represented by the median rather than the mean, with the median less sensitive to outliers [31]. Chi-Square tests were performed to evaluate between group differences for the categorical data (non-attendance rate, satisfaction and global improvement scores). Independent-samples t-test was performed to assess between-group differences in number of physiotherapy consultations with data reported as mean \pm SD.

\section{Results}

\section{Participant flow and recruitment}

Figure 1 illustrates the flow of participants during the study. Of 116 patients deemed suitable for ED Physiotherapy, 78\% ( $n=91)$ opted for the telephone assessment and advice service. Of those deemed eligible at that stage, $40 \%(n=36)$ contacted the service; however three participants were excluded $(n=1$ did not consent; $n=2$ poor English). Table 1 illustrates baseline demographic and clinical characteristics for each group.

Table 1: Participant Demographics

\begin{tabular}{|l|l|l|}
\hline & \multicolumn{2}{|c|}{ No (\%) of patients } \\
\hline & Usual care (n=17, 17\%) & Telephone service (n=86, 83\%) \\
\hline Female sex & $6(35 \%)$ & $37(41 \%)$ \\
\hline Age (years) § & $48(17)$ & $45(17)$ \\
\hline Employed* & $9(53 \%)$ & $18(21 \%)$ \\
\hline Time off work (days) §* & $25(31)$ & $15(28)$ \\
\hline Site of musculoskeletal problem** & $3(18 \%)$ & $5(6 \%)$ \\
\hline Cervical & 0 & $1(1 \%)$ \\
\hline Thoracic & $1(6 \%)$ & $19(22 \%)$ \\
\hline Lumbar & $7(41 \%)$ & $15(17 \%)$ \\
\hline Upper Limb & $6(35 \%)$ & $42(49 \%)$ \\
\hline Lower Limb & 0 & $4(5 \%)$ \\
\hline Multiple & $6(5-8)$ & $4(2-16)$ \\
\hline Duration of symptoms (weeks)†* & $14(82 \%)$ & $20(61 \%)$ \\
\hline New presentation* & $3(18 \%)$ & $13(39 \%)$ \\
\hline Recurrent presentation* & \multicolumn{2}{|l}{} \\
\hline $\begin{array}{l}\text { §Mean (standard deviation) } \\
\text { † Median (interquartile range) } \\
\text { *Excludes those that did not opt in/attend initial appointment }\end{array}$ \\
\hline
\end{tabular}

\section{Primary and secondary outcomes}

Those that contacted the telephone assessment and advice service had a significantly reduced wait time for consultation (median 6 days; 3 - 8 days) compared to those that opted for the face to face care 
pathway (median 35 days; $19-39$ days) ( $p \leq 0.05)$.

For the telephone advice and assessment group, there was 99 appointments in total, with 10 'did-notattends' at subsequent face to face appointments, resulting in a $10 \%$ non-attendance rate. For the usual care group, there was 68 appointments in total, with 15 'did-not-attends', resulting in a $22 \%$ nonattendance rate. This difference was non-significant between both groups $\left(X^{2}(2)=4.41, p>0.05\right)$.

There was no statistically significant difference between groups with regards to overall satisfaction $\left(X^{2}(3)=3.44, p>0.05\right)$ (Figure $2 \&$ Figure 3 ).

There was no statistically significant difference between groups with regards to global improvement scores $\left(X^{2}(4)=3.00, p>0.05\right)$ (Figure 4$)$.

\section{Process of care}

Of the 33 eligible participants that contacted the telephone assessment and advice service, 14 (43\%) were managed entirely by telephone consultation. Patients in the telephone assessment and advice service also had fewer physiotherapy contacts overall (via telephone and face to face) (mean $2.7 \pm 2.4$ ) compared to the usual care group (mean $3.1 \pm 2.3)(p>0.05)$.

\section{Discussion}

Our findings show that, compared with usual face to face care, the telephone assessment and advice service care pathway was equally clinically effective and provided faster access to ED physiotherapy without compromising on service user satisfaction. This novel care pathway also appeared to be more cost-efficient with reduced non-attendance rates and fewer physiotherapy contacts, with more than a third being managed by telephone consultation alone. No other study to date has evaluated this service delivery method within an ED physiotherapy service, although the findings of this study concur with 'PhysioDirect' evaluation findings of reduced wait time, reduced non-attendance rate and fewer consultations within a primary care setting when a physiotherapy telephone service is used [12].

This telephone assessment and advice service operated via a 'one-way' system in general, where the senior physiotherapist waited for patients to call them at specified times on a Monday, Wednesday and Friday morning. These times were chosen based on previous service provision and to minimise unnecessary delays to service access as much as possible. Nevertheless, the median wait time was 6 days, in line with a similar study [12] which reported a median of 7 days although slightly higher than the 3.55 days reported for a self-referral only telephone triage service [32]. Since longer waiting times are associated with an increase in non-attendance rates [33], this likely explains the reduced non-attendance rate observed in this study for the telephone assessment and advice service compared to usual care, which had a median wait time of 35 days, in line with waiting time figures for hospital outpatient physiotherapy services in Canada [34,35] and the UK [36]. 
Findings from this study suggest that more than a third of those suitable for an ED Physiotherapy service can be managed via telephone consultation alone. This figure is somewhat lower than figures reported in similar studies $(47-50 \%)[12,15]$ although it is worth noting that the former study results are based on telephone consultations by nurses in a primary care setting and this may partially explain this discrepancy. In some instances within an ED physiotherapy setting, face-to-face consultations may be indicated to comprehensively screen for potentially serious pathology with a patients' clincial presentation not always falling into a clear diagnositc category [14]. A high level of agreement between telehealth (specifically videoconferencing) and face-to-face assessments with regards to clinical management decisions has been demonstrated [37]; however this finding is not generalisable to acute and subacute musculoskeletal presentations commonly encountered within an ED physiotherapy setting. Limited response to the telephone-delivered intervention or patient preferences are other reasons why a face-to-face consultation was indicated.

This care pathway was designed in a patient centred manner; patients' chose the care pathway based on their preferences following a discussion with an ED referrer. It is worth noting that $78 \%$ opted for the telephone assessment and advice service suggesting this eHealth solution is broadly acceptable to patients. Furthermore, there was no significant difference between groups with respect to the satisfaction survey results, with both groups demonstrating a similar response rate. This is in contrast to findings from the 'PhysioDirect' evaluation [12], which indicated that patients in the usual care arm had slightly higher satisfaction compared to those in the telephone assessment and advice arm with regards to overall satisfaction. This between group difference was small however ( 0.19 points on a six point scale ranging from 'very poor' to 'excellent'), with the authors questioning the meaningfulness of this result. Another explanation for this difference may be that our telephone assessment and advice service operated alongside a usual face to face care pathway, representing one method of accessing physiotherapy services rather than a replacement of face-to-face care pathways, in line with conclusions from qualitative research nested within the 'PhysioDirect' study that reported that many participants felt this service model was a useful option for accessing early physiotherapy advice for their musculoskeletal conditon [19].

This study found that there were no differences between the telephone assessment and advice group and the usual face to face group with regards to clinical effectiveness, measured in terms of overall improvement levels. One possible explanation for this finding is that only one measure, a global improvement score was utilised to characterise clinical effectiveness. While this measure has good psychometric properties across a broad range of musculoskeletal conditons [38], it is insufficent to comprehensively evaluate this multidimensional concept. It is worth nothing Salisbury et al. [12] found similar results within the primary care setting utilsing five measures of clinical outcome in the 'PhysioDirect' evaluation. Another possible explanation may be that evidence-based guidelines for a number of musculoskeletal conditions recommends exercise and self-management interventions which can be effectively delivered remotely $[13,39,40]$. 
A number of limitations are acknowledged, with the cohort study design, high number (60\%) that did not contact the telephone advice and assessment service, along with the imbalance between the numbers undertaking each pathway, being key elements that limit the strength of the findings. While the 'did not contact' figure is not dissimilar from the 'PhysioDirect' evaluation, we do not have data about whether people did not contact as their symptoms had improved or perhaps they had changed their mind about the eHealth service. Another limitation is that the results about patient satisfaction only pertain to those that completed their physiotherapy episode of care and do not include those lost to follow-up. Finally, concerns about sensitivity of generic clinical improvement measures exist and while one was utilised from a feasibility point of view as no one disease specific measure was appropriate, this may have an impact on our findings

\section{Conclusion}

In conclusion, this study indicates that a telephone assessment and advice service can be a safe, efficient and effective means of reducing delays for advice for musculoskeletal problems for patients referred by a member of an ED team for ED Physiotherapy. This eHealth option appeared to be broadly acceptable to patients without compromising on clinical effectiveness. However, given the relatively small sample, and use of simple generic outcome measures, further research involving a larger population is warranted to validate these findings.

\section{Abbreviations}

ED: $\quad$ Emergency Department

iPIMS: Implement Single Patient Administration System

SPSS: $\quad$ Statistical Package for the Social Sciences Version

\section{Declarations}

\section{Funding}

This research did not receive any specific grant from funding agencies in public, commercial, or not-forprofit sectors.

\section{Competing interests}

The authors declare to have no financial affiliation or interest in any topic included in this manuscript. The authors declared that they have no conflict of interest.

\section{References}


[1] James SL, Abate D, Abate KH, Abay SM, Abbafati C, Abbasi N, et al., Global, regional, and national incidence, prevalence, and years lived with disability for 354 diseases and injuries for 195 countries and territories, 1990-2017: a systematic analysis for the Global Burden of Disease Study 2017, The Lancet. 2018:392(10159)1789-1858.

[2] Nations U, World population ageing: highlights, United Nations New York, 2017.

[3] Group NEMPW, The national emergency medicine programme, 2012.

[4] Zigenfus GC, Yin J, Giang GM, Fogarty WT, Effectiveness of early physical therapy in the treatment of acute low back musculoskeletal disorders, Journal of occupational and environmental medicine. 2000:42(1)35.

[5] Mäntyselkä PT, Kumpusalo EA, Ahonen RS, Takala JK, Direct and indirect costs of managing patients with musculoskeletal pain-challenge for health care, European journal of pain. 2002:6(2)141-148.

[6] Consulting J, A Survey of NHS Physiotherapy Waiting Times, Workforce and Caseloads in the UK 2010-2011, Chartered Society of Physiotherapy (PD090) London, 2011.

[7] Deslauriers S, Déry J, Proulx K, Laliberté M, Desmeules F, Feldman DE, et al., Effects of waiting for outpatient physiotherapy services in persons with musculoskeletal disorders: a systematic review, Disability and Rehabilitation. 2019:1-10. https://doi.org/10.1080/09638288.2019.1639222.

[8] Salisbury C, Foster NE, Bishop A, Calnan M, Coast J, Hall J, et al., 'PhysioDirect'telephone assessment and advice services for physiotherapy: protocol for a pragmatic randomised controlled trial, BMC Health Services Research. 2009:9(1)136.

[9] Gatchel RJ, Polatin PB, Noe C, Gardea M, Pulliam C, Thompson J, Treatment- and cost-effectiveness of early intervention for acute low-back pain patients: A one-year prospective study, Journal of Occupational Rehabilitation. 2003:13(1)1-9. <Go to ISI>://WOS:000180184800001.

[10] Wand BM, Bird C, McAuley JH, Doré CJ, MacDowell M, De Souza LH, Early intervention for the management of acute low back pain: a single-blind randomized controlled trial of biopsychosocial education, manual therapy, and exercise, Spine. 2004:29(21)2350-2356.

https://www.cochranelibrary.com/central/doi/10.1002/central/CN-00527659/full.

[11] Otto L, Harst L, Schlieter H, Wollschlaeger B, Richter P, Timpel P, Towards a Unified Understanding of eHealth and Related Terms-Proposal of a Consolidated Terminological Basis, HEALTHINF, 2018, pp. 533539.

[12] Salisbury C, Montgomery AA, Hollinghurst S, Hopper C, Bishop A, Franchini A, et al., Effectiveness of PhysioDirect telephone assessment and advice services for patients with musculoskeletal problems: pragmatic randomised controlled trial, BMJ (Clinical research ed.). 2013:346(f43. 
[13] Hinman RS, Campbell PK, Lawford BJ, Briggs AM, Gale J, Bills C, et al., Does telephone-delivered exercise advice and support by physiotherapists improve pain and/or function in people with knee osteoarthritis? Telecare randomised controlled trial, British journal of sports medicine. 2019.

[14] Greenhalgh S, Finucane LM, Mercer C, Selfe J, Safety netting; best practice in the face of uncertainty, Musculoskeletal Science and Practice. 2020:102179.

[15] Bunn F, Byrne G, Kendall S, Telephone consultation and triage: effects on health care use and patient satisfaction, Cochrane Database of Systematic Reviews. 2004:3).

[16] Lattimer V, George S, Thompson F, Thomas E, Mullee M, Turnbull J, et al., Safety and effectiveness of nurse telephone consultation in out of hours primary care: randomised controlled trial, BMJ (Clinical research ed.). 1998:317(7165)1054-1059.

[17] Lattimer V, Sassi F, George S, Moore M, Turnbull J, Mullee M, et al., Cost analysis of nurse telephone consultation in out of hours primary care: evidence from a randomised controlled trial, BMJ (Clinical research ed.). 2000:320(7241)1053-1057.

[18] Pinnock H, Bawden R, Proctor S, Wolfe S, Scullion J, Price D, et al., Accessibility, acceptability, and effectiveness in primary care of routine telephone review of asthma: pragmatic, randomised controlled trial, BMJ (Clinical research ed.). 2003:326(7387)477.

[19] Pearson J, Richardson J, Calnan M, Salisbury C, Foster NE, The acceptability to patients of PhysioDirect telephone assessment and advice services; a qualitative interview study, BMC health services research. 2016:16(1)104.

[20] Frost H, Lamb SE, Doll HA, Carver PT, Stewart-Brown S, Randomised controlled trial of physiotherapy compared with advice for low back pain, BMJ (Clinical research ed.). 2004:329(7468)708.

[21] Rivero-Arias O, Gray A, Frost H, Lamb SE, Stewart-Brown S, Cost-utility analysis of physiotherapy treatment compared with physiotherapy advice in low back pain, Spine. 2006:31(12)1381-1387.

[22] Lamb SE, Gates S, Williams MA, Williamson EM, Mt-Isa S, Withers EJ, et al., Emergency department treatments and physiotherapy for acute whiplash: a pragmatic, two-step, randomised controlled trial, The Lancet. 2013:381(9866)546-556.

[23] Foster NE, Thomas E, Barlas P, Hill JC, Young J, Mason E, et al., Acupuncture as an adjunct to exercise based physiotherapy for osteoarthritis of the knee: randomised controlled trial, BMJ (Clinical research ed.). 2007:335(7617)436.

[24] Hay EM, Foster NE, Thomas E, Peat G, Phelan M, Yates HE, et al., Effectiveness of community physiotherapy and enhanced pharmacy review for knee pain in people aged over 55 presenting to primary care: pragmatic randomised trial, BMJ (Clinical research ed.). 2006:333(7576)995. 
[25] Smidt N, de Vet HC, Bouter LM, Dekker J, Effectiveness of exercise therapy: a best-evidence summary of systematic reviews, Australian Journal of Physiotherapy. 2005:51(2)71-85.

[26] Çelik D, Kaya Mutlu E, Does adding mobilization to stretching improve outcomes for people with frozen shoulder? A randomized controlled clinical trial, Clinical rehabilitation. 2016:30(8)786-794.

[27] Miller J, Gross A, D'Sylva J, Burnie SJ, Goldsmith CH, Graham N, et al., Manual therapy and exercise for neck pain: a systematic review, Manual therapy. 2010:15(4)334-354.

[28] Page MJ, Green S, Kramer S, Johnston RV, McBain B, Chau M, et al., Manual therapy and exercise for adhesive capsulitis (frozen shoulder), Cochrane Database of Systematic Reviews. 2014:8).

[29] Vandenbroucke JP, von Elm E, Altman DG, Gøtzsche PC, Mulrow CD, Pocock SJ, et al., Strengthening the Reporting of Observational Studies in Epidemiology (STROBE): explanation and elaboration, International journal of surgery. 2014:12(12)1500-1524.

[30] IBM Corp. Released 2015. IBM SPSS Statistics for Windows, Version 23.0. Armonk, NY: IBM Corp.

[31] D'Addio G, Cesarelli M, Corbi G, Romano M, Furgi G, Ferrara N, et al., Reproducibility of heart rate turbulence indexes in heart failure patients, 2010 Annual International Conference of the IEEE Engineering in Medicine and Biology, IEEE, 2010, pp. 2573-2576.

[32] Mallett R, Bakker E, Burton M, Is Physiotherapy Self-Referral with Telephone Triage Viable, Costeffective and Beneficial to Musculoskeletal Outpatients in a Primary Care Setting?, Musculoskeletal care. 2014:12(4)251-260.

[33] Holdsworth LK, Webster VS, McFadyen AK, Group SPSRS, Are patients who refer themselves to physiotherapy different from those referred by GPs? Results of a national trial, Physiotherapy. 2006:92(1)26-33.

[34] Deslauriers S, Raymond M-H, Laliberté M, Lavoie A, Desmeules F, Feldman DE, et al., Access to publicly funded outpatient physiotherapy services in Quebec: waiting lists and management strategies, Disability and rehabilitation. 2017:39(26)2648-2656.

[35] Passalent LA, Landry MD, Cott CA, Wait times for publicly funded outpatient and community physiotherapy and occupational therapy services: implications for the increasing number of persons with chronic conditions in Ontario, Canada, Physiotherapy Canada. 2009:61(1)5-14.

[36] CSP, Chartered Society of Physiotherapy. Physiotherapy out- patient services survey 2012. London, UK: Contract No.: PD103., 2013.

[37] Cottrell MA, O'Leary SP, Swete-Kelly P, Elwell B, Hess S, Litchfield M-A, et al., Agreement between telehealth and in-person assessment of patients with chronic musculoskeletal conditions presenting to 
an advanced-practice physiotherapy screening clinic, Musculoskeletal Science and Practice. 2018:38(99105.

[38] Kamper SJ, Ostelo RW, Knol DL, Maher CG, de Vet HC, Hancock MJ, Global Perceived Effect scales provided reliable assessments of health transition in people with musculoskeletal disorders, but ratings are strongly influenced by current status, Journal of clinical epidemiology. 2010:63(7)760-766. e1.

[39] Dario AB, Cabral AM, Almeida L, Ferreira ML, Refshauge K, Simic M, et al., Effectiveness of telehealthbased interventions in the management of non-specific low back pain: a systematic review with metaanalysis, The Spine Journal. 2017:17(9)1342-1351.

[40] Pastora-Bernal JM, Martín-Valero R, Barón-López FJ, Estebanez-Pérez MJ, Evidence of benefit of telerehabitation after orthopedic surgery: a systematic review, Journal of medical Internet research. 2017:19(4)e142.

\section{Figures}




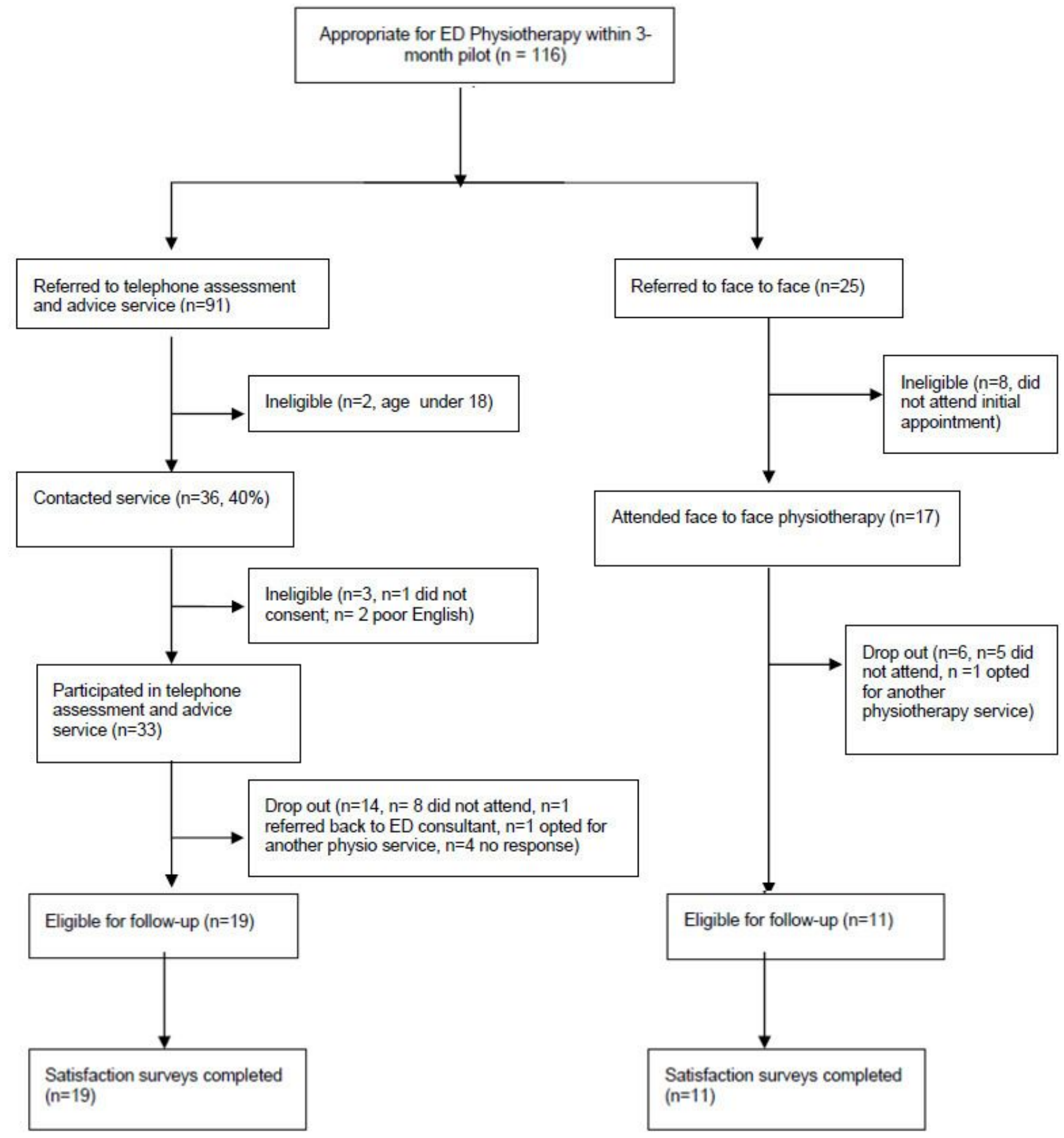

\section{Figure 1}

Flow of Participants through the study 


\section{Satisfaction data - face-to-face group $(n=11)$}

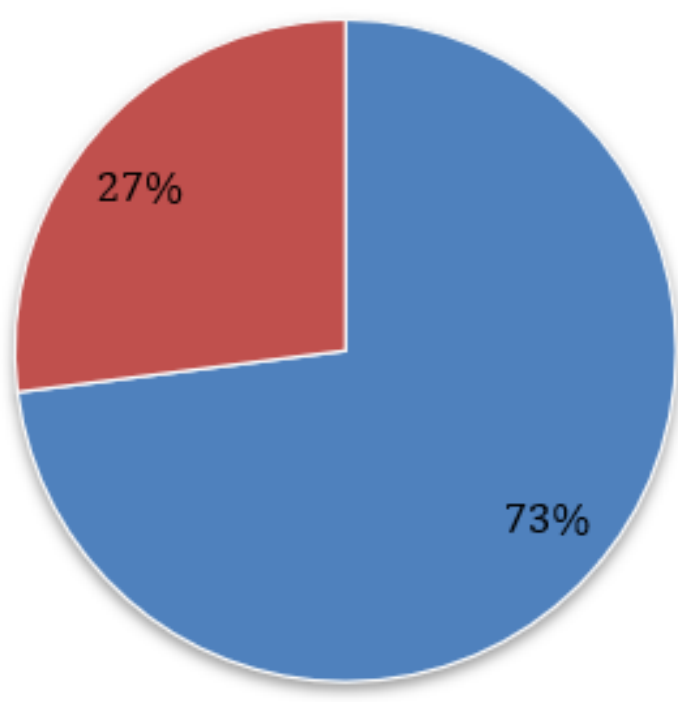

- Very Satisfied

- Fairly Satisfied

Figure 2

Global improvement scores for both groups 


\section{Satisfaction data - telephone assessment $\&$ advice group $(n=19)$}

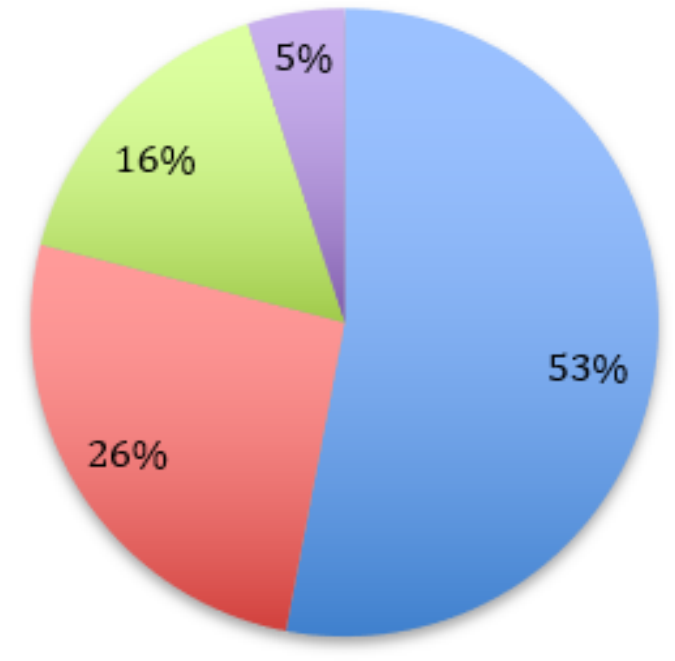

- Very satisfied

Fairly satisfied

Neither satisfied nor dissatisfied

- Fairly dissatisfied

Figure 3

Satisfaction data results for telephone assessment \& advice service $(n=19)$ 


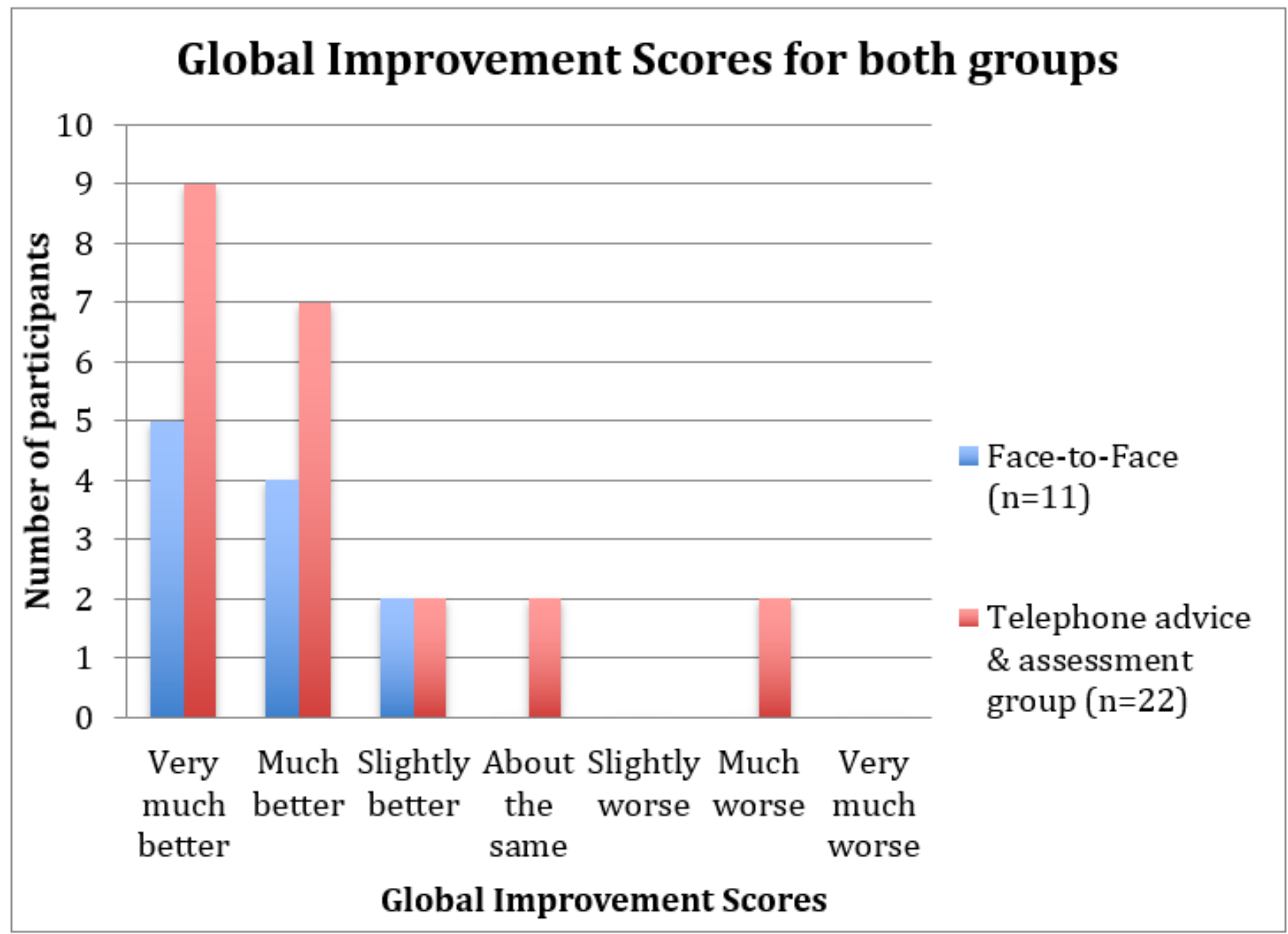

Figure 4

Global improvement scores for both groups 